\title{
The Application of Wireless Sensor in Aquaculture Water Quality Monitoring
}

\author{
Wen Ding and Yinchi Ma* \\ Beijing Fisheries Research Institute, Beijing, 100068, China \\ \{dingwen, mayinchi\} abjfishery.com
}

\begin{abstract}
The current means for aquaculture water quality monitoring have a weak infrastructure. We research to use wireless sensor technology, embedded computing technology, MEMS technology, distributing information processing technology and wireless communication technology to build the wireless network sensor network system. This system is a digital, networked, intelligent real-time dynamic for monitoring the aquaculture water quality. The system not only can deal the normal detection of the aquaculture environment indicators (temperature, $\mathrm{PH}$, dissolved oxygen, turbidity, ammonia, etc.) in real-time monitoring, but also can detect indicators of data fusion and data mining to establish a history database of aquaculture environmental monitoring indicators. The system can gather the monitoring data by a local or remote way, and realize the real-time, dynamic display and analysis. So as to improve the process of aquaculture, water resources utilization, the quality of the culture environment and reduce emissions of pollutants. The system can provide an important technical means and scientific basis. The hardware architecture design is the core technology of this system. We will detail the part in this article.
\end{abstract}

Keywords: wireless sensor network, node, gateway, hardware platforms, aquaculture applications.

\section{$1 \quad$ Introduction}

Wireless sensor network is a kind of wireless network without infrastructure. It integrates the sensor technology, the embedded computing technology, the modern network technology, the wireless communication technology and the distributed intelligent information processing technology. The system can work under a state of long-term unattended [1]. It can detect, perceive and gather the information of the monitoring or environmental objects within the networks distribution region in real-time. It receives and sends messages through wireless and self-organization multi-hop routing [2]. Merging the logical information world and the real physical world together will change the interaction way between people and nature. The technology has good prospects in many areas, especially in agriculture area. Wireless sensor networks will be able to play a significant role in environmental monitoring, precision agriculture, section irrigation and livestock breeding [3-5].

${ }^{*}$ Corresponding author. 
This study is using the wireless sensor network technology to build an intelligent, network-based wireless monitoring system for aquaculture environment. The technology can be quickly applied to the field of aquaculture environmental monitoring, and promote the research and application of the wireless sensor networks technology. So as to improve the current traditional farming methods and aquaculture environmental monitoring instruments in the field of aquaculture in China. In this paper, we propose a set of practical hardware architecture design for the application of aquaculture.

\section{System Design}

The system mainly consists of the multi-parameter water quality sensors, the digital-analog transmission module, the wireless sensor network, the GPRS transmission unit, the data query and messaging real-time analysis of early warning system, and several other components. As shown in Figure 1, a set of wireless real-time water quality monitoring system includes the data acquisition node, the relay node and the gateway node.

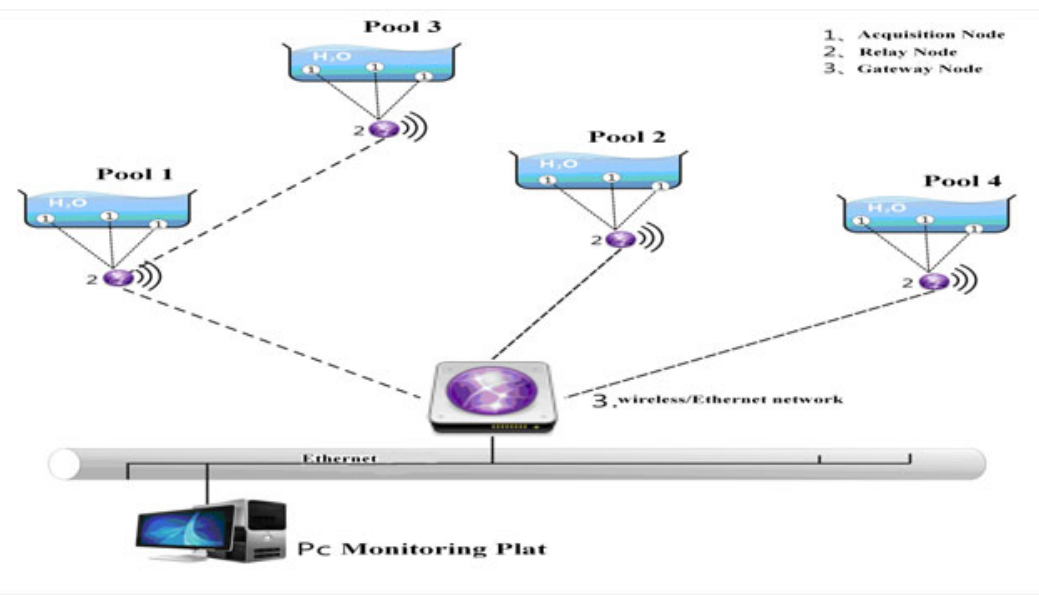

Fig. 1. Hardware architecture

\section{Hardware Implementation}

\subsection{Acquisition Node}

Monitoring can be arbitrarily placed in the desired area. Real-time collection includes water temperature, $\mathrm{PH}$, dissolved oxygen, turbidity, ammonia, etc., and transmit the collected data to a wireless repeater or wireless gateway by wireless way. 


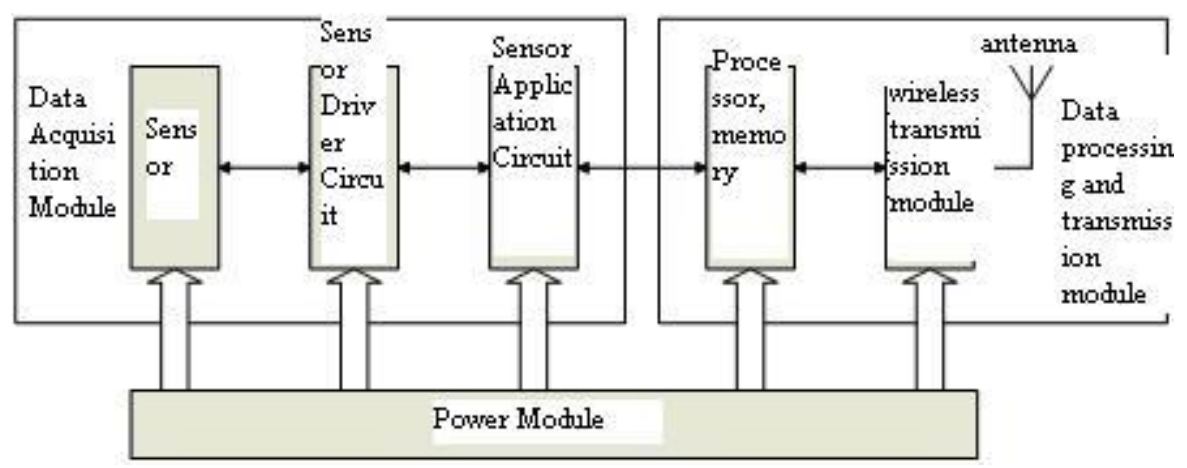

Fig. 2. Data Acquisition Node

As the figure 2 shows, the data acquisition node is an integrated structure. And it mainly contains the data acquisition module and the data transmission module, including the following several units divided by function:

\section{- Sensor module}

Sensor module contains the sensors, the sensor drive circuit and the sensors applied circuit. Sensors including the water temperature sensor, the $\mathrm{PH}$ value sensor, the oxygen sensor, the turbidity sensor, the ammonia nitrogen sensor for the water environment monitoring of the aquaculture water.

- Data processing and transmission module

I. Processor and memory unit: including the processor, the memory and the I/O. Among them, the processor is the low-energy micro controller.

II. Wireless transmitting/receiving module unit: the unit is composed of the wireless communication module according with IEEE802.15.4 / Zigbee. And this unit is used for the data wireless transmission and communication.

\section{- Power module}

Power supply unit is the power and control section of the system. The most important characteristic of the sensor node is that it will work under the condition of no lasting permanent power supply. This feature requires the work energy necessary of the sensor node is very low, and the sustainable power supply time of its own power must be long. According to the different application, appropriately adjusting the sleep time of sensor is the main task of the power control. Usually, the AA batteries and lithium battery can maintain a sensor node survival several months to 2 years. And adopting new technologies, such as solar energy, or using air micro vibration to produce electric power can make a sensor node survive longer. In the system, the scheme adopts the rechargeable efficient lithium battery.

\subsection{Relay Node}

The main function of relay nodes are forwarding sampling data from sensor node, and transmitting to gateway or other relay through wireless self-organization network. It 
can realize the transmission with a much longer distance. Relay node completely realized self-organizing network routing algorithms, within the wireless communication region, the relay nodes can immediately join in the network after powering, and establish the route of the data transmission.

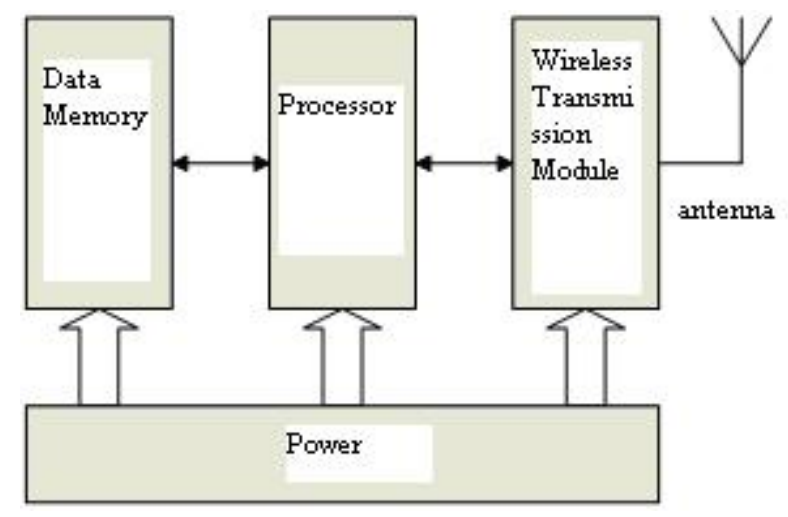

Fig. 3. Relay Node

As the figure 3 shows, the relay node mainly contains the data processing and wireless transmission modules with the same function as the data acquisition node. Among them according to their function, including the following several units divided by function:

- Data processing and transmission module

I. Processor and memory unit: including the processor, the memory and the I/O. Among them, the processor is the low-energy micro controller.

II. Wireless transmitting/receiving module unit: the unit is composed of the wireless communication module according with IEEE802.15.4 / Zigbee. And this unit is used for the data wireless transmission and communication.

\section{- Data storage module}

Data storage module is mainly composed of RAM or FLASH memory unit, and its main function is to store all kinds of sensor data and preserve the routing information of the whole network.

\subsection{Gateway Node}

The acquisition data by sensor node is transmitted to PC monitoring platform through Ethernet gateway. The wireless network/Ethernet gateway has wireless sensor networks, IEEE802.3 Ethernet and GPRS communication function. It can transfer the wireless communication data to the Ethernet protocol and the GPRS data. It can put any wireless input data transparent transmission to a server with designated IP address, and the maximum data throughput can achieve 22.5 Kbytes/s. The RFIC adopt CC1100 of Chipcon Company. The processor adopts Mega128L of Atmel Company. The maximum 
visual transmission distance can be more than $800 \mathrm{~m}$. The transmission distance of a typical aquaculture site is more than $300 \mathrm{~m}$, and it supports IEEE802.3 Ethernet networks, GPRS wireless transmission, and TCP/IP, UDP protocol transparent data transmission.

Data communication can be set up through the Ethernet gateway, wireless networks and any other PC in Ethernet. Gateway IP address, address masking and communication port can be online set through gateway set software. PC monitoring software exchanges data with gateway according to IP address and communication port. Gateway will sent sensor network data to with conventional format.

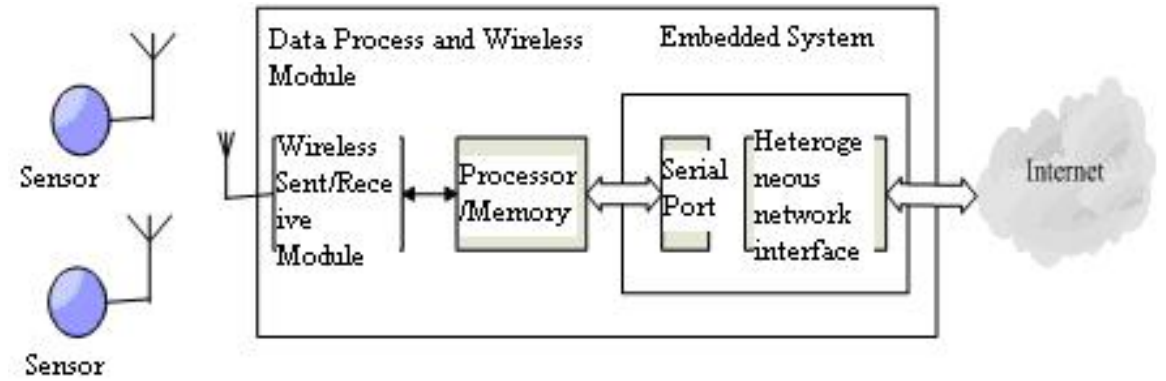

Fig. 4. Gateway Node

As the Figure 4 shows, the gateway node contains the data processing and wireless transmission module with the same function as the data acquisition node, and the embedded system connected with the heterogeneous network. As a IEEE802.15.4 and sensor network communication agent, the sensor node function modules must be equipped with a external I/O communication unit, used to communicate with the agency application server (Ethernet, RS232, USB, GPRS and satellite communication, etc.). The data from the sensor network accepted by the gateway node will be processed by the application server with stronger calculating function, but without power limit.

\section{Conclusion}

This study constructs an intelligent, networked aquaculture environment monitoring system using wireless sensor network technology. And the system is applied in the aquaculture environment monitoring field. It promotes the level of our wireless sensor network technology development and application. Ant it provides a new technical solution for improving the backward traditional breeding way and aquaculture environment monitoring method of the Chinese aquaculture field.

The low power consumption, low cost, and high reliability characteristics of the wireless sensor network make it have a broad application prospect in the wireless remote environment testing field [6 - 8]. We put forward the aquaculture water quality monitoring system construction program based on the study of Zigbee protocol of the wireless sensor network technology. And the water quality parameters collection and analysis was finished on this platform. 
The results show that the development of the all-weather "wireless intelligent network real-time monitoring and warning system for aquaculture environment " can realize the real-time automatic monitoring, remote wireless transmission, automatic data processing and analysis, multi-platform control, intelligent warning, and SMS alarm for the water temperature, dissolved oxygen, $\mathrm{PH}$, turbidity, ammonia and other indicator parameters of aquaculture environmental. So as to improve the utilization of aquaculture water maximum, provide the appropriate environmental condition for aquaculture objects, and control and ensure the quality security of aquatic products effectively. The system can realize automatic monitoring and scientific management for the aquaculture environment during the whole process. It will play a important role for protecting high-yield, efficient, safe, health of intensive aquaculture, improving aquatic product quality and safety in our city, and promoting the intensive, industrialization, refinement of aquaculture, and realizing the sustainable development of Beijing urban agriculture.

\section{References}

1. Wu, J.: Design of Wireless Sensor Network Node for Water Quality Monitorin. Computer Measurement \& Control (12) (2009)

2. $\mathrm{Du}, \mathrm{Z}$.: Design of water quality monitoring wireless sensor network system based on wireless sensor. Computer Engineering and Design (17) (2008)

3. Liu, X.: Aquaculture security guarantee system based on water quality monitoring and its application. Transactions of the Chinese Society of Agricultural Engineering (6) (2009)

4. Ma, C., Ni, W.: The design of a factory aquiculture monitor system based on PLC. Industrial Instrumentation \& Automation (02) (2005)

5. Zhu, W., Ran, G.: Research of aquaculture environment parameter automatic monitoring and controlling system. Freshwater Fisheries (01) (2001)

6. Guo, S., Ma, S., Wu, P.: The application research of remote monitoring system based on Zigbee technology of wireless sensor network in family. Application of Electronic Technique (6), 28-30 (2006)

7. Ren, X., Yu, H.: The wireless sensor network safety analysis based on ZigBee technology. Computer Science 6(10), 111-113 (2001)

8. Zhang, H., Li, W.: Study of a Wireless Sensor Network Based on ZigBee Technology. Journal of Wuhan University of Technology (Information \& Management Engineering) (8), $12-15$ (2006) 